\title{
The Effects of Sex on Patient Reported Outcomes in Inflammatory Arthritis and Connective Tissue Diseases*
}

\author{
Jason J. Lee ${ }^{1}$, Janet E. Pope ${ }^{2 \#}$ \\ ${ }^{1}$ Schulich School of Medicine, Western University, London, Canada; ${ }^{2}$ Schulich School of Medicine, Western University, St. Joseph’s \\ Health Care, London, Canada. \\ Email: \#janet.pope@sjhc.london.on.ca
}

Received April $1^{\text {st }}, 2013$; revised May $2^{\text {nd }}, 2013$; accepted May $9^{\text {th }}, 2013$

Copyright (c) 2013 Jason J. Lee, Janet E. Pope. This is an open access article distributed under the Creative Commons Attribution License, which permits unrestricted use, distribution, and reproduction in any medium, provided the original work is properly cited.

\begin{abstract}
Background: It was thought that women report higher pain than men. We studied if there was a sex difference for several patient reported outcomes (PROs) in rheumatic diseases. Materials and Methods: Health Assessment Questionnaire disability index (HAQ-DI) as well as $100 \mathrm{~mm}$ Visual Analogue Scale (VAS) for pain, fatigue, sleep disturbance, and patient global assessment were compared cross-sectionally between the sexes for ankylosingspondylitis (AS), psoriatic arthritis (PsA), rheumatoid arthritis (RA), systemic lupus erythematosus (SLE) and systemic sclerosis (SSc). Data were collected using standardized forms administered during routine care. Results: The sample included 136 patients (97 males) with AS, 200 (83 males) with PsA, 232 (40 males) with RA, 199 (12 males) with SLE, and 113 (17 males) with SSc. There were no significant differences in AS. There were sex differences in PsA for HAQ $(0.85$ females, 0.57 males; $\mathrm{p}<0.003$ ), pain (45.2 females, 36.8 males; $\mathrm{p}<0.03$ ), sleep (42.1 females, 31.6 males; $\mathrm{p}<0.025$ ), and not significantly for fatigue and global scores (fatigue: 44.4 females, 36.0 males; $\mathrm{p}<0.07$. global: 40.1 females, 33.1 males; $\mathrm{p}$ $<0.06$ ). There were similar non-significant differences observed in RA and SLE; whereas, in SSc, men had a higher global assessment (52.9 males, 38.1 females; $\mathrm{p}<0.03$ ). Conclusions: A significant sex difference was observed in PsA with females reporting worse symptoms. In SSc, global assessments were worse in males possibly due to proportionately more diffuse cutaneous SSc. Sex differences for PROs are not consistent between rheumatic inflammatory diseases in prevalent patients.
\end{abstract}

Keywords: Sex Differences; Patient Reported Outcomes; Inflammatory Arthritis; Connective Tissue Disease

\section{Introduction}

The effects of inflammatory arthritis and connective tissue diseases have high impact on patients including pain, fatigue, functional loss, and work loss [1-6]. As these groups of patients with chronic disease grow in incidence and prevalence, it is increasingly more important to better evaluate and understand these patients. A good example of such an initiative is a recent epidemiological study from the Mayo Clinic in Minnesota, USA, which concluded that the incidence of rheumatoid arthritis (RA), one of the most common forms of inflammatory arthritis, is increasing, particularly in women [7].

Several studies that have explored the effects of sex on patient reported outcomes (PROs) in inflammatory arthritis have focused on pain in RA [1,2,6,8]. Disease ac-

\footnotetext{
*There was no funding for this project. There are no conflicts of interest. Ethics approval was obtained for the chart audit.

"Corresponding author.
}

tivity measured by Disease Activity Score 28 (DAS28) (which includes a patient global assessment), and self reported functional impairment as measured by the Health Assessment Questionnaire Disability Index (HAQ-DI) [9] were significantly higher in females compared to males. In ankylosing spondylitis (AS), there are sex differences in phenotype where females may present with less severe disease, but have more peripheral arthritis [4]. The authors in the 2007 study found that males with AS present with worse radiographic axial disease. However, females reported worse function despite less severe objective measures of disease. This could have been confounded perhaps by more peripheral arthritis.

The distributions of males compared to females vary between rheumatic diseases [1,2,4,6,7,10-14]. It is highest in AS with often 7 to 9 males to 1 female [4,10], to approximately equal in PsA [11], female predominance in RA $[1,2,6]$ (approximately $75 \%$ to $80 \%$ female in RA 
RCTs) and greater than 8:1 females to males in SSc [13] and SLE [14]. The differences in disease manifestations between males and females could potentially affect PROs.

PROs are often used in intervention studies so recognizing potential sex related differences may help in the interpretation of and generalizability to patients in rheumatologists' practices. Currently, it is widely believed that females suffer more pain from their arthritis. Data is sparse in the rheumatology literature for potential sex differences in other PROs such as fatigue or sleep disturbance. Sex differences in PROs in other inflammatory rheumatic conditions such as psoriatic arthritis (PsA), systemic lupus erythematosus (SLE) and systemic sclerosis (SSc) have not been as well studied.

We compared HAQ-DI, pain, fatigue, sleep disturbance, and patient reported global disease activity between sexes for AS, PsA, RA, SLE, and SSc to determine if each disease has sex effects on patient reported outcomes. We hypothesized that women would report worse outcomes across all inflammatory arthritis and CTDs.

\section{Patients and Methods}

Multiple data are collected routinely on patients seen at St. Joseph's Hospital Rheumatology clinic in London, Ontario, which is affiliated with Western University, and services a referral region of over one million. The clinic is heavily weighted towards seeing patients with inflammatory arthritis and CTDs. Patients are primarily English speaking, representing the full spectrum of disease severity, and are generally followed 1 - 4 times a year depending on disease severity and flare-ups. The databases from previous studies of minimally important differences (MID) were used in AS, PsA, RA, SLE and SSc where patients seen consecutively who completed their clinic forms and were seen in follow up in less than one year were included [10-14]. These publications had Ethics Approval from the University of Western Ontario Ethics Committee for studying RA, SLE, SSc, PsA, AS, and Sjogren's syndrome and this current project utilized the data collected under the approval which covered this project also.

The data are from patients seen serially by one rheumatologist (JP) who had been diagnosed with RA [15] and SLE [16] meeting the ACR criteria, diagnosed with PsA meeting the Moll and Wright criteria [17], and diagnosed with SSc by expert opinion, most of whom met the ACR criteria [18], or having the limited cutaneousSSc subset who did not meet criteria (as $12 \%$ of patients with SSc in the limited subset may not meet ACR criteria) [19]. The data for AS are from patients seen serially by five rheumatologists in the clinic who had been diagnosed with AS as per ACR criteria [20]. The patients are referred from the community and patients with rare diseases such as scleroderma are often referred from a larger catchment area.

Mean and distribution of scores for consecutive patients with these diseases were recorded. Patient data were collected during routine visits, as part of usual patient care. For analysis, data points were collected for two consecutive visits that were 6 to 18 months apart. Patient global status was defined and measured with the following question: "How would you describe your overall status since your last visit: much better, better, the same, worse, much worse". HAQ-DI was scored as 0 (no disability) to 3 (severe disability/limitation in function), and VAS for pain, fatigue, sleep and global health ( 0 as no problem and 100 as worst). Changes of $10 \%$ or approximately $10 \%$ on a $100 \mathrm{~mm}$ visual analogue scale (VAS) correspond to MID for patient reported measures across many studies [21].

Data for each disease were extracted by a single trained data-extractor and entered into a common database. From these databases, one author (JL) extracted relevant clinical and demographic data using Microsoft Excel and performed the necessary statistical analyses. PROs from a cross sectional follow-up visit were used for analysis. Two-tailed distribution T-tests were done to determine if there were significant sex differences for PROs in each disease. Data were analyzed using SPSS v. 19.0. A two-tailed $\mathrm{p}<0.05$ was considered significant. Results were presented as mean (SD) unless if otherwise specified.

\section{Results}

A total of 880 patients were included for this observational study. Descriptive demographic statistics are summarized in Table 1. The breakdown of patient groups according to disease were as follows: 136 (29\% female) with AS, 200 patients (59\% female) with PsA, 232 patients (83\% female) with RA, 199 patients (94\% female) with SLE and 113 patients (85\% female) with SSc. Patients' age and duration of disease were similar between men and women as well as across all diseases. The average age in years for men and women respectively were as follows: AS: 44 (11.5), 45 (12.2); PsA: 51 (14.7), 51 (13.5); RA: 62 (11.3), 58 (14.7); SLE: 56 (10.1), 52 (14.3); and SSc: 53 (10.2), 58 (11.6). The average duration of disease was long (10 to 16 years) (Table 1).

There were no significant differences for PROs in AS. There were differences in PsA for HAQ ( 0.85 females, 0.57 males; $\mathrm{p}<0.003$ ), pain (45.2 females, 36.8 males; $\mathrm{p}$ $<0.034$ ), and sleep (42.1 females, 31.6 males; $\mathrm{p}<0.025$ ) where females had worse scores. There were non-significant higher scores observed in women with RA and SLE, except in HAQ-DI within SLE where there was a significant difference ( 0.65 females, 0.20 males; $\mathrm{p}<0.001$ ). 
Table 1. Study patient demographics.

\begin{tabular}{ccccccccccc}
\hline $\begin{array}{c}\text { Patient } \\
\text { Demographics }\end{array}$ & $\begin{array}{c}\text { Ankylosing Spondylitis } \\
(\mathrm{N}=136)\end{array}$ & $\begin{array}{c}\text { Psoriatic Arthritis } \\
(\mathrm{N}=200)\end{array}$ & $\begin{array}{c}\text { Rheumatoid Arthritis } \\
(\mathrm{N}=232)\end{array}$ & $\begin{array}{c}\text { Systemic Lupus } \\
\text { Erythematosus } \\
(\mathrm{N}=199)\end{array}$ & $\begin{array}{c}\text { Systemic Sclerosis } \\
(\mathrm{N}=113)\end{array}$ \\
\hline & Male & Female & Male & Female & Male & Female & Male & Female & Male & Female \\
$\mathrm{N}(\%)$ & $97(71 \%)$ & $39(29 \%)$ & $83(41 \%)$ & $117(59 \%)$ & $40(17 \%)$ & $192(83 \%)$ & $12(6 \%)$ & $187(94 \%)$ & $17(15 \%)$ & $96(85 \%)$ \\
$\begin{array}{c}\text { Mean Age (SD) } \\
\begin{array}{c}\text { Mean Disease } \\
\text { Duration (SD) }\end{array}\end{array}$ & $15.8(10.7)$ & $14.6(10.3)$ & $12.3(9.2)$ & $10.4(6.7)$ & $10.6(11.2)$ & $11.8(10.5)$ & $12.6(8.9)$ & $10.0(7.2)$ & $9.8(7.1)$ & $9.9(6.5)$ \\
$\begin{array}{c}\text { Median Duration } \\
(\text { min, max })\end{array}$ & $12(1,45)$ & $13(1,35)$ & $11(1,60)$ & $10(1,36)$ & $6(1,45)$ & $8(1,57)$ & $10(1,30)$ & $8(1,40)$ & $7(1,29)$ & $10(1,35)$ \\
\hline
\end{tabular}

Age and disease duration in years; SD = Standard Deviation

The opposite occurred between males and females within SSc where men scored higher, but only the global assessment was significantly different (52.9 males, 38.1 females; $\mathrm{p}<0.035)$. The PROs are shown in Figure 1.

\section{Discussion}

The incidence and prevalence of inflammatory arthritis and connective tissue diseases may be increasing [7]. The evolving epidemiology of the diseases may include changes in prognosis and can influence treatment [1,2,4,6,7]. One important, but under-explored, observation is the effects of sex on disease symptom severity and associated clinical impact. Understanding PROs are important because they are widely used and can influence treatment decisions.

Earlier studies in the 1980s, before the discovery modern disease modifying anti-rheumatic drugs (DMARDs), reported poor outcomes in many patients without appreciable sex differences [3,5]. However, more recent studies in the 1990s and 2000s with more effective therapeutic agents and early aggressive treatment strategies have discovered major differences between sexes with respect to various disease outcomes $[1,2,6]$. Currently, it is widely believed that there are significant sex differences in objective measures of disease such as remission rates, age of onset, and production of biologic markers for common rheumatic diseases, particularly in RA [2,6]. There is evidence of worse pain and associated disability in women with RA and PsA [1,2,6,11].

Contrary to these recent findings, in our patient cohort, consistent sex differences were not observed for patient reported outcome measures. Only females with PsA reported marginally significant worse symptoms compared to males. Of note, in our RA patients, the PROs differed from other RA cohorts reported in literature $[1,2,6]$. There was lower pain compared to Ahlmen et al. [1] who reported pain VAS of approximately 47in men over a 5-year span, and approximately 43 in women during the same time interval. In comparison, pain VAS scores were 38.5 and 38.7 for men and women in our patients. It is possible that, while women and men may respond to therapies differently, contemporary DMARD treatment with early intervention and use of combination therapies may alter sex differences for PROs in RA. Interestingly, in the most recent meta-analysis by Barnabe et al. [2], only 3 of 16 cohorts of patients with RA reported mean pain VAS of less than $40 \mathrm{~mm}$ for both men and women. Of these three cohorts, 2 of 3 did not find significance in the difference between men and women.

In SSc males numerically had higher scores, although not statically significant for most of the PROs that were studied. We believe that this is likely due to males having proportionately more diffused subset of SSc (dcSSc) with worse disease severity than localized scleroderma (lcSSc) [13], but we did not have the power to divide the subsets for this study.

The study has limitations. It consists of patients with long disease duration seen cross-sectionally and there could have been differences in disease activity and treatment as objective disease measures were not studied. However, it is unlikely that treatment would be biased by gender. The study could have been underpowered; especially where there were small numbers for certain subsets by sex within a disease. For instance, some p-values may have been significant if numbers were larger. The proportion of males in each group, with the exception of AS, was smaller than females, which is consistent with epidemiological studies [2,4,6,8,10-14,19]. The different proportions of males in each disease could also impact on comorbidities that may have different proportions between the sexes and comorbidities can affect patient reported outcomes. For instance, fibromyalgia is a common comorbidity in women with SLE. We did not take comorbidities into account in this study. The patients were from a single clinic and, with exception of the AS group, from a single rheumatologist. The patient population was somewhat homogeneous with most patients being Caucasian, able to read English. Also, the long disease duration (mean 10 - 16 years) would affect patient scores (adaptation over time and more damage over time) so the results 


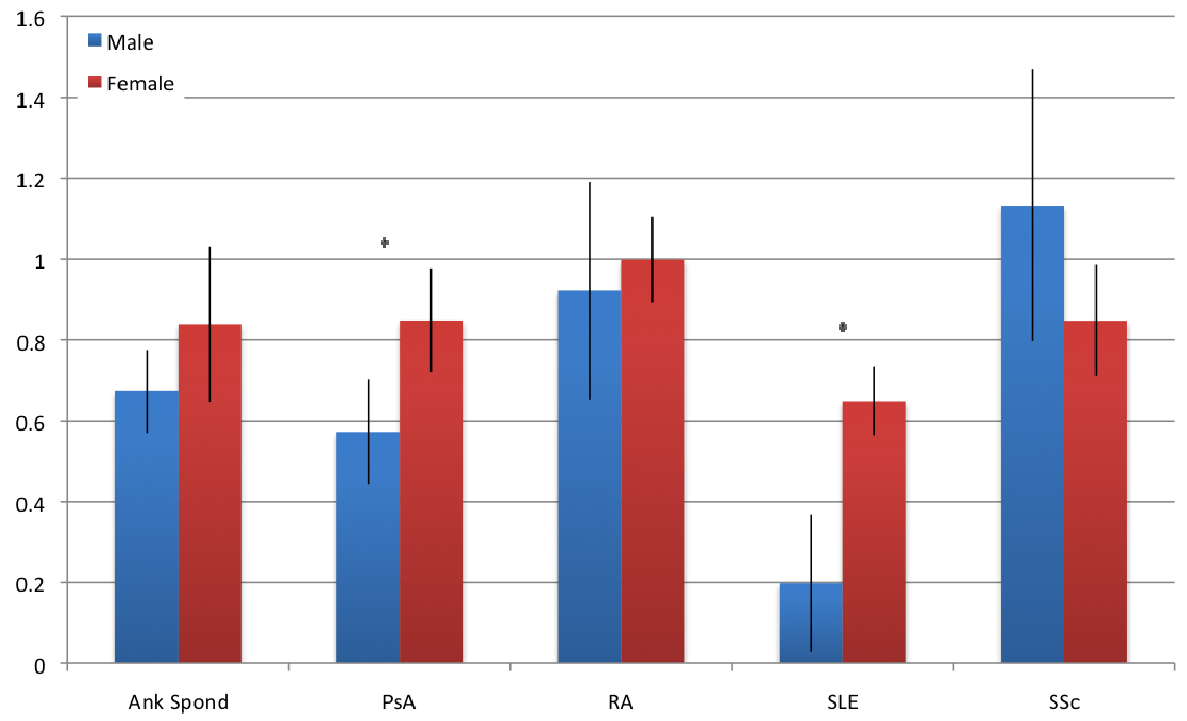

(a)

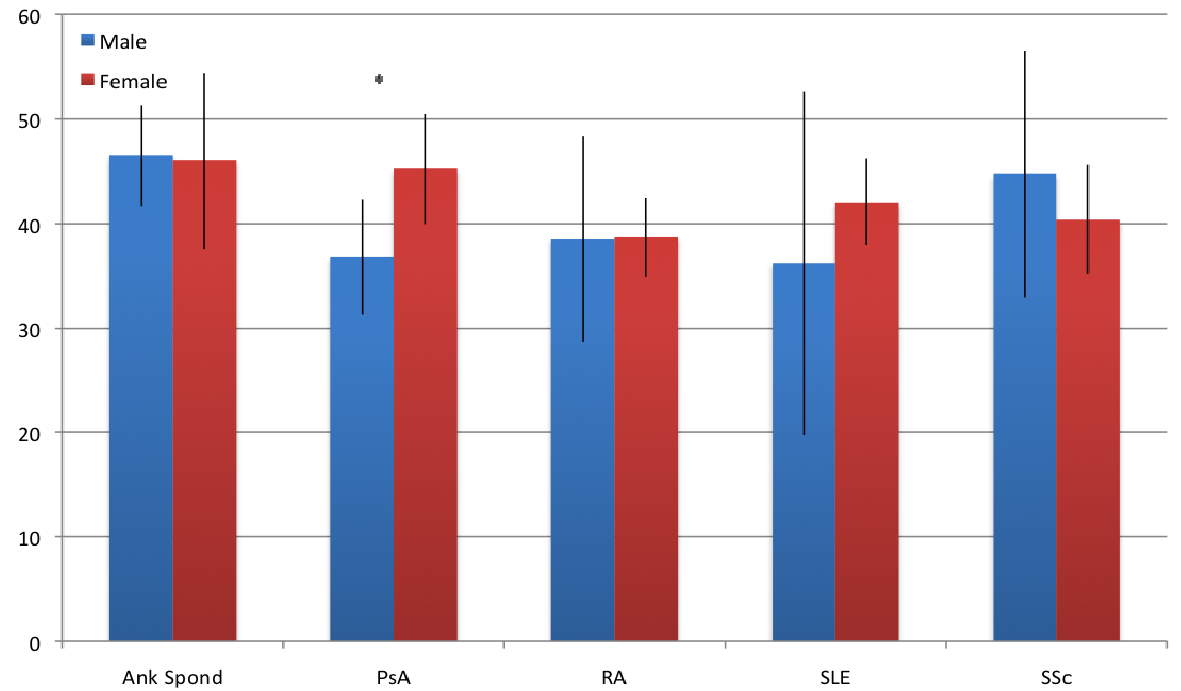

(b)

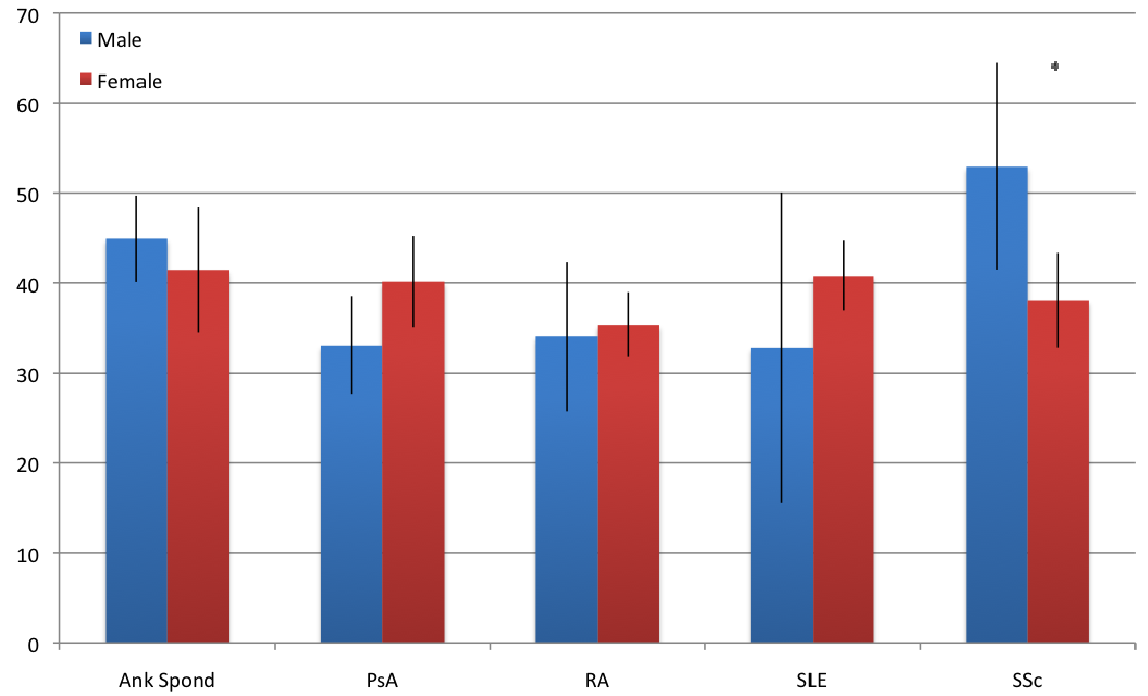

(c) 


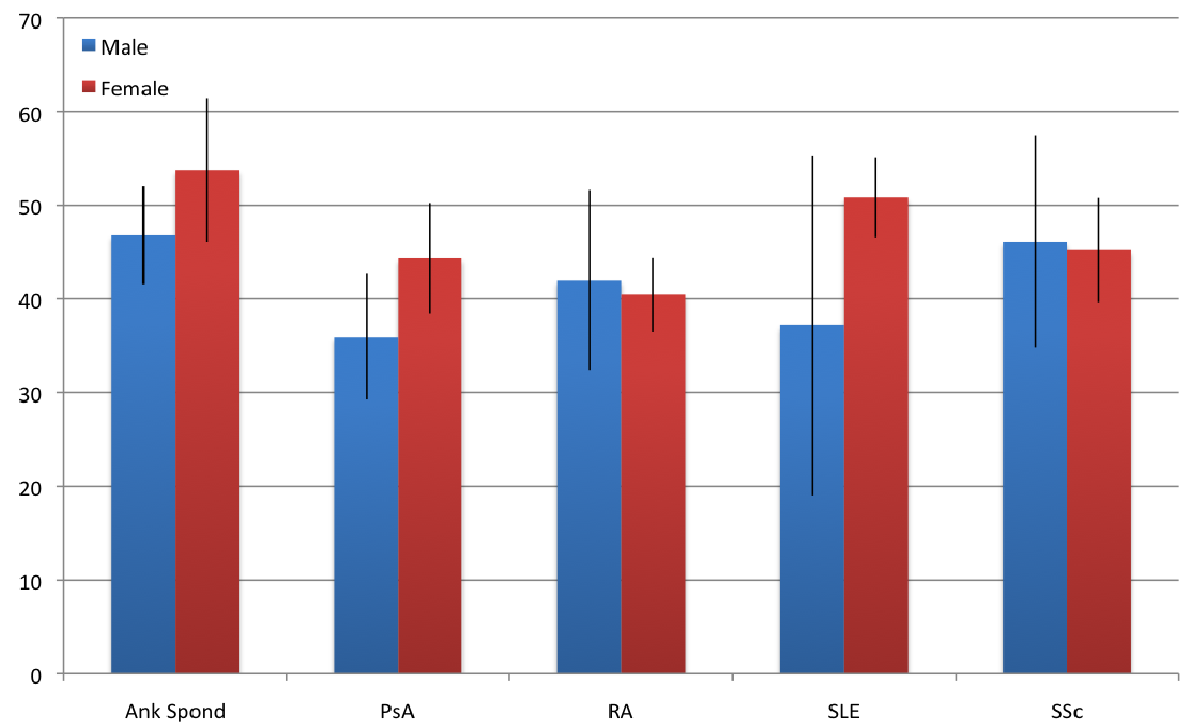

(d)

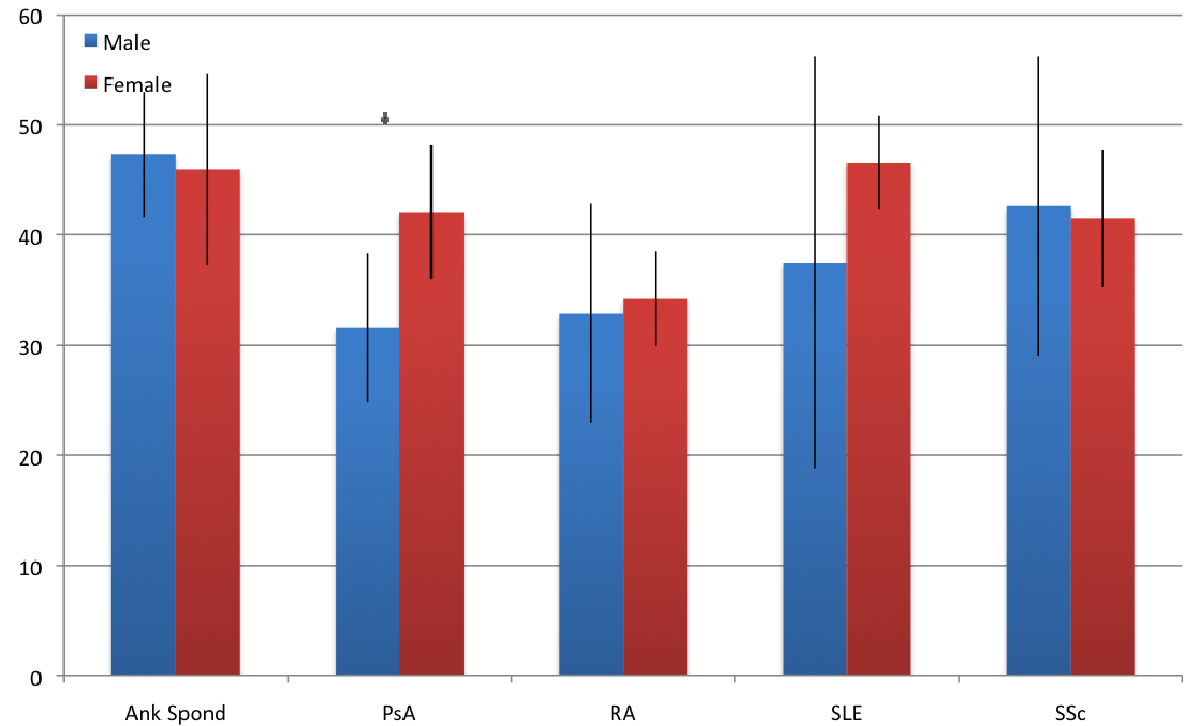

(e)

Figure 1. Summary of the means for all patient reported outcome measures: (a) Health assessment questionnaire disability index; (b) Pain visual analog scale in $\mathrm{mm}$; (c) Global visual analog scale in $\mathrm{mm}$; (d) Fatigue visual analog scale in mm; (e) Sleep visual analog scale in $\mathbf{m m}$. The error bars represent $95 \%$ confidence intervals and $*$ Indicates significance with $\mathrm{p}<0.05$.

may not be generalizable to early disease. The data presented here were collected at a single visit. Although this has the potential for bias, data analyzed within the same patients from a subsequent follow-up usually within 6 to 12 months were similar (data not shown). Multiple statistical testing was not corrected using Bonferroni's as the entire study was a secondary hypothesis as data were used from previous MCID studies. It may be that disease activity is affecting the differences in PROs more than gender but we did not have all variables for disease activity consistently collected on the patients included in this study. It may be that sex affects treatment which then would influence the PROs (such as a disparity between use of biologics in inflammatory arthritis).

\section{Conclusion}

In summary, this is the first study to comprehensively characterize sex differences for several inflammatory rheumatologic diseases for many PROs. Mostly there were no sex differences. However, a significant sex difference was observed in PsA, with females reporting worse symptoms. In SSc patient global assessment was worse in males. Sex differences for patient reported outcomes are not consistent between rheumatic inflammatory diseases. 


\section{REFERENCES}

[1] M. Ahlmen, B. Svensson, K. Albertsson, K. Forslind and I. Hafström, "Influence of Gender on Assessments of Disease Activity and Function in Early Rheumatoid Arthritis in Relation to Radiographic Joint Damage,” Annals of the Rheumatic Diseases, Vol. 69, No. 1, 2010, pp. 230-233.

[2] C. Barnabe, L. Bessette, C. Flanagan, S. Leclercq, A. Steiman, F. Kalache, et al., "Sex Differences in Pain Scores and Localization in Inflammatory Arthritis: A Systematic Review and Metaanalysis," The Journal of Rheumatology, Vol. 39, No. 6, 2012, pp. 1221-1230. doi:10.3899/jrheum.111393

[3] K. Kaarela, "Prognostic Factors and Diagnostic Criteria in Early Rheumatoid Arthritis," Scandinavian Journal of Rheumatology, Vol. 14, No. s57, 1985, pp. 1-54. doi:10.3109/03009748509104317

[4] W. Lee, J. D. Reveille, J. C. J. Davis, T. J. Learch, M. M. Ward and M. H. Weisman, "Are There Gender Differences in Severity of Ankylosing Spondylitis? Results from the PSOAS Cohort," Annals of Rheumatic Diseases, Vol. 66, No. 5, 2007, pp. 633-638. doi:10.1136/ard.2006.060293

[5] G. Makisara and P. Makisara, "Prognosis of Functional Capacity and Work Capacity in Rheumatoid Arthritis," Clinical Rheumatology, Vol. 1, No. 2, 1982, pp. 117-125. doi:10.1007/BF02275601

[6] T. Sokka, S. Toloza, M. Cutolo, H. Kautiainen, H. Makinen, F. Gogus, et al., "Women, Men, and Rheumatoid Arthritis: Analyses of Disease Activity, Disease Characteristics, and Treatments in the QUEST-RA Study,” Arthritis Research \& Therapy, Vol. 11, No. 1, 2009, p. R7.

[7] E. Myasoedova, C. S. Crowson, H. M. Kremers, T. M. Therneau and S. E. Gabriel, "Is the Incidence of Rheumatoid Arthritis Rising? Results from Olmsted County, Minnesota, 1955-2007,” Arthritis \& Rheumatism, Vol. 62, No. 6, 2010, pp. 1576-1582. doi:10.1002/art.27425

[8] D. Khanna, J. E. Pope, P. P. Khanna, M. Maloney, N. Samedi, D. Norrie, et al., "The Minimally Important Difference for the Fatigue Visual Analog Scale in Patients with Rheumatoid Arthritis Followed in an Academic Clinical Practice,” The Journal of Rheumatology, Vol. 35, No. 12, 2008, pp. 2339-2343. doi:10.3899/jrheum.080375

[9] J. Fries, P. Spitz, R. Kraines and H. Holman, "Measurement of Patient Outcome in Arthritis," Arthritis \& Rheumatism, Vol. 23, No. 2, 1980, pp. 137-145. doi:10.1002/art.1780230202

[10] L. Wheaton and J. Pope, “The Minimally Important Difference for Patient-Reported Outcomes in Spondyloarthropathies Including Pain, Fatigue, Sleep, and Health Assessment Questionnaire," The Journal of Rheumatology, Vol. 37, No. 4, 2010, pp. 816-822. doi:10.3899/jrheum.090086

[11] T. Kwok and J. E. Pope, "Minimally Important Difference for Patient-Reported Outcomes in Psoriatic Arthritis:
Health Assessment Questionnaire and Pain, Fatigue, and Global Visual Analog Scales," The Journal of Rheumatology, Vol. 37, No. 5, 2010, pp. 1024-1028. doi:10.3899/jrheum.090832

[12] J. Pope, D. Khanna, D. Norrie and J. M. Ouimet, “The Minimally Important Difference for the Health Assessment Questionnaire in Rheumatoid Arthritis Clinical Practice Is Smaller than in Randomized Controlled Trials," The Journal of Rheumatology, Vol. 36, No. 2, 2009, pp. 254-259. doi:10.3899/jrheum.080479

[13] S. Sekhon, J. Pope, Canadian Scleroderma Research Group and M. Baron, "The Minimally Important Difference in Clinical Practice for Patient-Centered Outcomes Including Health Assessment Questionnaire, Fatigue, Pain, Sleep, Global Visual Analog Scale, and SF-36 in Scleroderma," The Journal of Rheumatology, Vol. 37, No. 3, 2010, pp. 591-598. doi:10.3899/jrheum.090375

[14] K. J. Colangelo, J. Pope and C. Peschken, “The Minimally Important Difference for Patient Reported Outcomes in Systemic Lupus Erythematosus Including the HAQ-DI, Pain, Fatigue, and SF-36," The Journal of Rheumatology, Vol. 36, No. 10, 2009, pp. 2231-2237. doi:10.3899/jrheum.090193

[15] F. C. Arnett, S. M. Edworthy, D. A. Bloch, D. J. McShane, J. F. Fries, N. S. Cooper, et al., "The American Rheumatism Association 1987 Revised Criteria for the Classification of Rheumatoid Arthritis,” Arthritis \& Rheumatism, Vol. 31, No. 3, 1988, pp. 315-324. doi:10.1002/art.1780310302

[16] M. C. Hochberg, "Updating the American College of Rheumatology Revised Criteria for the Classification of Systemic Lupus Erythematosus,” Arthritis \& Rheumatism, Vol. 40, No. 9, 1997, p. 1725. doi:10.1002/art.1780400928

[17] J. M. Moll and V. Wright, "Psoriatic Arthritis,” Seminars in Arthritis and Rheumatism, Vol. 3, No. 1, 1973, pp. 5578. doi:10.1016/0049-0172(73)90035-8

[18] T. Alfonse, "Preliminary Criteria for the Classification of Systemic Sclerosis (Scleroderma),” Arthritis \& Rheumatism, Vol. 23, No. 5, 1980, pp. 581-590. doi:10.1002/art.1780230510

[19] E. C. LeRoy, C. M. Black, R. Fleischmajer, et al., "Scleroderma (Systemic Sclerosis): Classification, Subsets and Pathogenesis," The Journal of Rheumatology, Vol. 15, No. 2, 1988, pp. 202-205.

[20] S. Van der Linden, H. A. Valkenburg and A. Cats, "Evaluation of Diagnostic Criteria for Ankylosing Spondylitis: A Proposal for Modification of the New York Criteria," Arthritis \& Rheumatism, Vol. 27, No. 4, 1984, pp. 361-368. doi:10.1002/art.1780270401

[21] G. R. Norman, J. A. Sloane and K. W. Wyrwich, "Interpretation of Changes in Health-Related Quality of Life: The Remarkable Universality of Half a Standard Deviation,” Medical Care, Vol. 41, No. 5, 2003, pp. 582-592. doi:10.1097/01.MLR.0000062554.74615.4C 